\title{
Circular RNAs Sparkle in the Diagnosis and Theranostics of Hepatocellular Carcinoma
}

\author{
Menglan Wang ${ }^{1}$, Minjie Wu ${ }^{1 *}$, Tian $\mathrm{Xie}^{1 *}$ and Jianxiang Chen ${ }^{1,2 *}$ \\ ${ }^{1}$ College of Pharmacy, School of Medicine, Department of Hepatology, Institute of Hepatology and Metabolic Diseases, \\ Institute of Integrated Chinese and Western Medicine for Oncology, The Affiliated Hospital of Hangzhou Normal University, \\ Key Laboratory of Elemene Class Anti-Cancer Chinese Medicines, Engineering Laboratory of Development and Application \\ of Traditional Chinese Medicines, Collaborative Innovation Center of Traditional Chinese Medicines of Zhejiang Province, \\ Hangzhou Normal University, Hangzhou, China, ${ }^{2}$ Laboratory of Cancer Genomics, Division of Cellular and Molecular \\ Research, National Cancer Centre Singapore, Singapore, Singapore
}

Exonic circular RNAs (circRNAs) are a novel subgroup of non-coding RNAs, which are generated by a back-splicing mechanism of the exons or introns. Unlike the linear RNA, circRNA forms a covalently closed loop, and it normally appears more abundant than the linear products of its host gene. Due to the relatively high specificity and stability of circular RNAs in tissues and body fluid, circular RNAs have attracted widely scientific interest for its potential application in cancer diagnosis and as a guide for preclinical therapy, especially for hard-to-treat cancers with high heterogeneity, such as hepatocellular carcinoma (HCC). Thus, we summarize the updated knowledge of circular RNAs, including the mechanism of the generation of endogenous circular RNAs and their regulatory, diagnostic, and therapeutic roles in HCC.

Keywords: HCC, circRNA, hepatocellular carcinoma, biomarker, therapy

\section{INTRODUCTION}

The regulation of the mammalian transcriptome is diverse and complex. In humans, only $2 \%$ of the human genome is transcribed into protein-coding RNAs, and approximately $95 \%$ transcripts are non-coding mRNAs (Esteller, 2011; Han et al., 2018). As early as the 1970s, it has been found that circular RNAs (circRNAs) exist in viruses and eukaryotic genome (Kolakofsky, 1976; Sanger et al., 1976; Hsu and Coca-Prados, 1979). Due to technical limitation, few circRNAs were identified at that time. In recent years, along with the advancement in the technology of deep sequencing, plenty of circRNAs have been identified in eukaryotes (Jeck et al., 2013; Salzman et al., 2013; Szabo et al., 2016; Li J. et al., 2020). Accumulating evidence indicates that circRNAs are involved in a series of physiological and pathological pathways, resulting in many diseases, notably in cancers. Hepatocellular carcinoma (HCC) is a malignant cancer affecting people's health globally. Due to the lack of a neural system in the liver, lesion of the liver in early stages is hard to be sensed because of a lack of symptoms. Mostly, patients with symptoms, like chest stuffiness and pain, are diagnosed at advanced stages with metastasis. The treatment outcome of late-stage HCC is dismal (Bray et al., 2018). The rapid lethality of late-stage HCC highlights the urgent need of early diagnosis and intervention (Zhou et al., 2018). CircRNAs consist of circular loops, which make them more stable and can be used as diagnostic markers (Qian et al., 2018). Due to the high abundance and stability and its unique expression signatures associated with cancer progression and prognosis, the 
potential of circRNAs acting as diagnostic and therapeutic biomarkers in HCC has been highlighted (Satoh and Yamamura, 2004; Hentze and Preiss, 2013; Filippenkov et al., 2017). In this review, we summarize the regulation of endogenous biosynthesis of circRNAs, their regulatory function and mechanisms in HCC, and the subsequent challenges and obstacles of applying circRNAs in clinical diagnosis and therapy.

\section{ENDOGENOUS BIOSYNTHESIS OF CIRCRNAS}

The mechanism of production of circRNAs remains elusive. The most widely accepted model is that circRNAs are derived by a back-splicing mechanism. Based on the diversity of splicing sequences, circRNAs are categorized into four types: exonic circRNAs (ecircRNAs), exon-intron circRNAs (EIciRNAs), intronic circRNAs (including ciRNAs derived from pre-mRNAs and tricRNAs derived from tRNA intronic circular RNAs), and intergenic circRNAs (Figure 1; Jeck et al., 2013; Memczak et al., 2013; Salzman et al., 2013; Zhang et al., 2013; Ashwal-Fluss et al., 2014; Li Z. et al., 2015; Wang et al., 2016). Similar with the canonical splicing regulation, the formation of backsplicing requires the canonical splicing signals and spliceosome (Ashwal-Fluss et al., 2014; Andres-Leon et al., 2016). However, the established regulators and mechanism of back-splicing are still largely unknown. By far, RNA-binding proteins (RBPs) have been identified as the potential regulatory factors for transacting circRNA splicing. There are several RBPs that have been identified, such as Muscleblind (MBL) (Ashwal-Fluss et al., 2014), Quaking (QKI) (Conn et al., 2015), adenosine deaminase acting on RNA 1 (ADAR1) (Ivanov et al., 2015), and DExH-box helicase 9 (DHX9) (Aktas et al., 2017). As for MBL and QKI, they can recognize the specific motifs within flanking introns and directly bind on them, dragging two splicing sites close enough to promote back-splicing subsequently (Ashwal-Fluss et al., 2014; Conn et al., 2015). Conversely, some RNPs impede circRNA formation by destroying the pairing of intronic elements (Ivanov et al., 2015; Aktas et al., 2017). For example, ADAR1 inhibits circRNA formation by binding to double-stranded RNA and melting the stem structure, thus generating ecircRNAs and EIciRNAs, and ciRNAs are limited in the nucleus, indicating their variable functions (Zhang et al., 2013; Li Z. et al., 2015). The most common formulation of circRNAs is via "back-splicing." There is a different way of RNA circularization occurring in archaea and eukaryotes, and circRNAs are derived from tRNA intron splicing (Figure 1; Noto et al., 2017).

\section{REGULATORY MECHANISMS OF CIRCRNAS ON BIOLOGICAL FUNCTIONS}

CircRNAs are involved in physiogenesis and pathogenesis due to their complex biological functions. They exert cellular functions mainly by regulating transcription, alternative splicing (AS), translating into peptides, and acting as protein decoy or scaffold or miRNA sponges (Table 1). Their distinct biological functions are discussed below.

\section{CircRNAs Regulate Transcription and Alternative Splicing}

As for different circRNAs, their locations in cells are quite different. EcircRNAs mainly exists in the cytoplasm, whereas EIciRNAs and ciRNAs mainly localize in the nucleus. EIciRNAs usually binds with U1 small nuclear ribonucleoprotein (U1 snRNP) via RNA-RNA interactions. The resultant complex further interacts with RNA polymerase II to enhance the transcription of ecircRNAs parental genes (Figure 2; Li Z. et al., 2015). Moreover, some circRNAs can compete with linear AS targets. For instance, circMbl competes with MBL pre-mRNA splicing during its formation (Ashwal-Fluss et al., 2014). Some circRNAs compete with linear splicing during transcription, leading to the production of circRNAs and linear mRNAs. Logically, due to the unfavorable assembly of spliceosomes at back-splicing sites, the efficiency of back-splicing is lower than canonical splicing. However, due to the damage of the core, premRNA processing components like splicing factor $3 \mathrm{~A}$ subunit 1 (SF3a1) and splicing factor 3B subunit 1 (SF3b1) are inhibited, leading to inhibited pre-mRNA splicing and enhanced backsplicing (Liang et al., 2017). Hence, the oscillation of circular and canonical AS could be a regulatory target for circRNA-mediated pathological activities (Zhang Y. et al., 2016; Liang et al., 2017; Vadlamudi et al., 2020).

\section{CircRNAs Translate Into Peptides}

Although circRNAs are categorized into non-coding RNAs, emerging evidence has indicated the potential role of circRNAs in protein translation (van Heesch et al., 2019). Through ribosomal profiling, exonic circRNAs that contain internal ribosome entry sites (IRES) or prokaryotic binding sites have been proven with potential protein-coding capacity. Moreover, bioinformatics analysis has been involved in the identification of the open reading frame (ORF) and the potential IRES on the sequence of circRNAs. It has been reported that the translation capability of circRNAs mainly depends on the IRES element or m6A modification recently discovered (Yang Y. et al., 2017; Figure 2). For instance, circ-ZNF609 (Legnini et al., 2017) has been confirmed with a protein translation role in myogenesis by a splicingdependent and cap-independent regulatory mechanism (Legnini et al., 2017). Moreover, the circMbl3-derived protein has been confirmed by mass spectrometry detection (Pamudurti et al., 2017). All the evidence above indicated a translational potential of circRNAs, which requires further exploration on their detailed regulatory mechanisms and their correlation with human diseases.

\section{CircRNAs Act as Protein Decoy or Scaffold}

It has been reported that circRNAs can act as a decoy or scaffold to sequester proteins and regulate gene expression or functional protein localization (Figure 2). For example, 


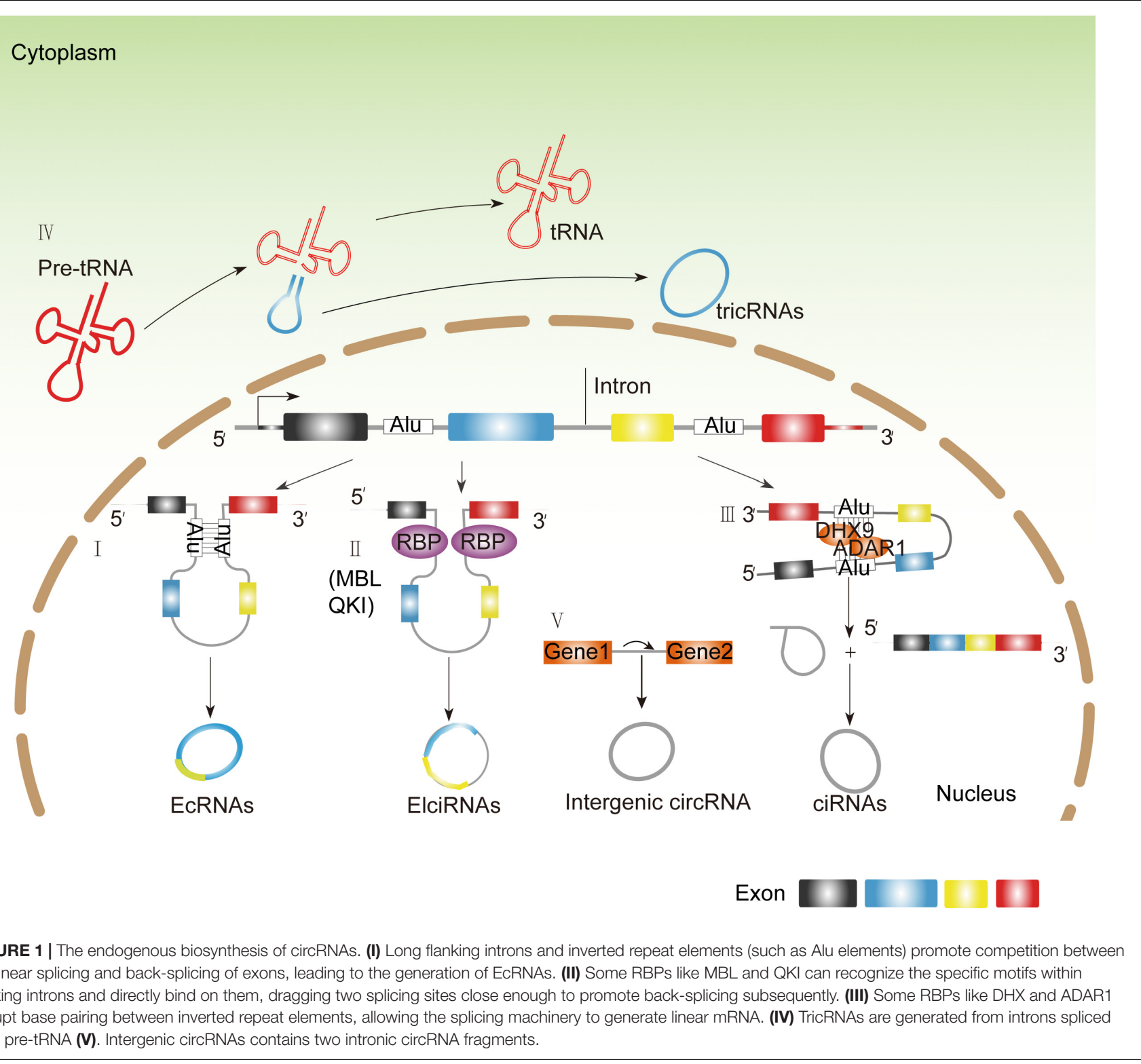

circPABPN1 and PABPN1 mRNA bind to HuR competitively to suppress the translation of PABPN1 (Abdelmohsen et al., 2017). CircFoxo3 inhibits the function of CDK2 via direct interaction with both CDK2 and p21 to form a ternary complex (Du et al., 2016). The interaction of circAmotl1 with AKT1 (Zeng et al., 2017), STAT3 (Yang Q. et al., 2017), c-myc (Yang Q. et al., 2017), and PDK1 (Zeng et al., 2017) alters their localization translocating from the cytoplasm to the nucleus, which further regulates their downstream target expression. In HCC, some RBP is involved in HCC progression. For instance, circZKSCAN1 negatively regulates cancer stem cells by physically binding FMRP against the CCAR1 complex in HCC. It exerts its inhibitive role by competitively binding with FMRP, therefore blocking the binding of FMRP and $\beta$-cateninbinding protein-cell cycle and apoptosis regulator 1 (CCAR1) mRNA and subsequently restraining the transcriptional activity of Wnt signaling (Zhu et al., 2019). Conclusively, the capability of circRNAs to interact with proteins may mainly depend on the nucleotide sequences and the secondary or tertiary structures of each circRNA, the detailed mechanism of which needs further precise study.

\section{CircRNAs Function as MiRNA Sponges}

To date, numerous reports have elucidated a common function of circRNAs as miRNA sponges. A large number of circRNAs localize in the cytoplasm, indicating their role in posttranscriptional regulation. The function of circRNAs as miRNA sponges was first identified in 2013, and the human circRNA running antisense to the Cerebellar DegenerationRelated protein 1 (CDR1) locus, termed as CDR1as, harbors about 70 conserved matches to the miR-7 seeds (Memczak et al., 2013). CDR1as also binds with AGO protein to enhance 
TABLE 1 | Regulatory mechanisms of circRNAs on biological functions.

\begin{tabular}{|c|c|c|}
\hline CircRNA & Model system & Function and mechanism \\
\hline circMbl & Neuronal tissues & $\begin{array}{l}\text { circMbl can compete with linear AS } \\
\text { targets (Ashwal-Fluss et al., 2014). }\end{array}$ \\
\hline circ-ZNF609 & Myoblast & $\begin{array}{l}\text { circ-ZNF609 has been confirmed with } \\
\text { protein translation role in myogenesis } \\
\text { by a splicing-dependent and } \\
\text { cap-independent regulatory } \\
\text { mechanism (Legnini et al., 2017). }\end{array}$ \\
\hline circMbl3 & $\begin{array}{l}\text { ribosome } \\
\text { footprinting from fly } \\
\text { heads }\end{array}$ & $\begin{array}{l}\text { the circMbl3-derived protein has been } \\
\text { confirmed by mass spectrometry } \\
\text { detection (Pamudurti et al., 2017). }\end{array}$ \\
\hline circPABPN1 & Cervical Carcinoma & $\begin{array}{l}\text { circPABPN1 and PABPN1 mRNA bind } \\
\text { to HuR competitively to suppress the } \\
\text { translation of PABPN1(Abdelmohsen } \\
\text { et al., 2017). }\end{array}$ \\
\hline CircFoxo3 & Non-cancer cells & $\begin{array}{l}\text { CircFoxo3 inhibits the function of CDK2 } \\
\text { via direct interaction with both CDK2 } \\
\text { and p21 to form a ternary complex (Du } \\
\text { et al., 2016). }\end{array}$ \\
\hline circAmotl1 & $\begin{array}{l}\text { neonatal human } \\
\text { cardiac tissue }\end{array}$ & $\begin{array}{l}\text { The interaction of circAmotl1 with } \\
\text { AKT1, STAT3, c-myc and PDK1 alters } \\
\text { their localization translocating from } \\
\text { cytoplasm to nucleus, which further } \\
\text { regulates their downstream targets } \\
\text { expression (Yang Q. et al., 2017; Zeng } \\
\text { et al., 2017). }\end{array}$ \\
\hline circZKSCAN1 & $\mathrm{HCC}$ & $\begin{array}{l}\text { circZKSCAN1 negatively regulates } \\
\text { cancer stem cells by physically binding } \\
\text { FMRP against CCAR1 complex (Zhu } \\
\text { et al., 2019). }\end{array}$ \\
\hline circSLC8A1 & $\begin{array}{l}\text { Bladder Cancer } \\
\text { Cardiac } \\
\text { Hypertrophy }\end{array}$ & $\begin{array}{l}\text { circSLC8A1 acts as a sponge for } \\
\text { miR130b/484 in bladder cancer (Lim } \\
\text { et al., 2019) and a sponge of miR-133 } \\
\text { in cardiac hypertrophy (Lu et al., 2019). }\end{array}$ \\
\hline
\end{tabular}

the expression of miR-7 targets (Hansen et al., 2013). Some circRNA serves as sponges for different miRNAs. For instance, circSLC8A1 acts as a sponge for miR130b/484 in bladder cancer (Lim et al., 2019) and a sponge of miR-133 in cardiac hypertrophy (Lu et al., 2019).

\section{THE ROLE OF CIRCRNAS IN HUMAN HEPATOCELLULAR CARCINOMA}

By far, numerous evidences have suggested that dysregulation of circRNAs is closely correlated with cancer initiation and/or progression. According to their roles in HCC progression, they can be classified into oncogenes, tumor suppressors, and circRNAs that interfere with hepatitis virus infection (Table 2). The other cancer-related roles of circRNAs were recently indicated in drug resistance and heterogeneity [Roles of circRNAs in the tumor microenvironment, Molecular Cancer volume 19, Article number: 14 (2020)], which is remarkable and widely detected in human HCC. Thus, we aim to summarize the circRNAs recently reported in the regulation of HCC. Due to space limitations, we apologize to the authors for the literature not listed and discussed here.

\section{CircRNAs Function as Oncogenes in HCC}

There have been numerous circRNAs identified in HCC, related with the promotion of cancer cells' proliferation and invasion, inhibition of apoptosis, and enhancement of angiogenesis. For instance, circRNA_100338 functions as a sponge of miR-141$3 p$ to promote invasion of HCC cells. The expression of circRNA_100338 is positively correlated with poorer of OS and PFS of HCC patients (Huang et al., 2017). circ_0005075 promotes HCC progression via miR-431 (Li et al., 2018), and exosomal circ-ZNF652 could transfer to HCC cells to promote cell proliferation, migration, invasion, and glycolysis in HCC via the miR-29a-3p/GUCD1 axis (Li Y. et al., 2020). CircMAST1 elicits HCC progression by sponging miRNA1299 and stabilizing CTNND1. CircMAST1 is upregulated in HCC tissues and cell lines; silencing circMAST1 with small interfering RNA inhibits the migration, invasion, and proliferation of HCC (Yu X. et al., 2020). CircZNF609 enhances HCC cell proliferation, metastasis, and stemness by activating the Hedgehog pathway through the regulation of miR-15a5p/15b-5p and GLI2 expressions (He et al., 2020). Exosome circdeubiquitination (circ-DB) is upregulated in HCC patients with higher body fat ratios. Exo-circ-DB promotes HCC growth and reduces DNA damage via suppressing miR-34a expression and activating deubiquitination-related USP7 (Zhang et al., 2019). Circ_0091579 promotes HCC progression by enhancing cell migration and invasion and impeding cell apoptosis (Jiang et al., 2020). hsa_circ_0000711 promotes proliferation and inhibits the apoptosis of hepatoma cells via targeting has-miR-103a-3p (Chen K. H. et al., 2020). Hsa_circ_104566 promotes HCC progression by decreasing apoptosis and E-cadherin, thus increasing cell viability, proliferation, migration, invasion, and $\mathrm{N}$-cadherin (Liu et al., 2020). Exosomal circRNA-100338 promotes HCC metastasis by enhancing invasiveness and angiogenesis (Huang et al., 2020). hsa_circ_0000092 competitively binds to miR-338$3 \mathrm{p}$ to upregulate HN1 expression, promoting angiogenesis in HCC (Pu et al., 2020). Circular RNA ciRS-7 (Cdrlas) acts as a risk factor of hepatic microvascular invasion in HCC (Xu et al., 2017). This evidence suggested that circRNAs play important roles in HCC progression via regulating angiogenesis.

\section{CircRNAs Act as Tumor Suppressors in HCC}

Conversely, there are some circRNAs that have been identified as tumor suppressors. circZKSCAN1 inhibits Wnt signaling to impede cancer stemness property and malignancy in HCC (Zhu et al., 2019). circ-MTO1 suppresses HCC progression by acting as a sponge of oncogenic miR-9 to promote p21 expression (Han et al., 2017). Expression of circ-MTO1 is negatively correlated with the prognosis of HCC patients (Han et al., 2017). cSMARCA5 inhibits the growth and migration of HCC by sponging of $\mathrm{miR}-17-3 \mathrm{p}$ and $\mathrm{miR}-181 \mathrm{~b}-5 \mathrm{p}$ to promote the expression of TIMP3, a well-known tumor suppressor (Yu et al., 2018). Circular RNA circ-102,166 acts as a sponge of miR-182 and miR-184 to suppress HCC proliferation and invasion (Li R. et al., 2020). CircRNA-ITCH inhibits cell proliferation and promotes 


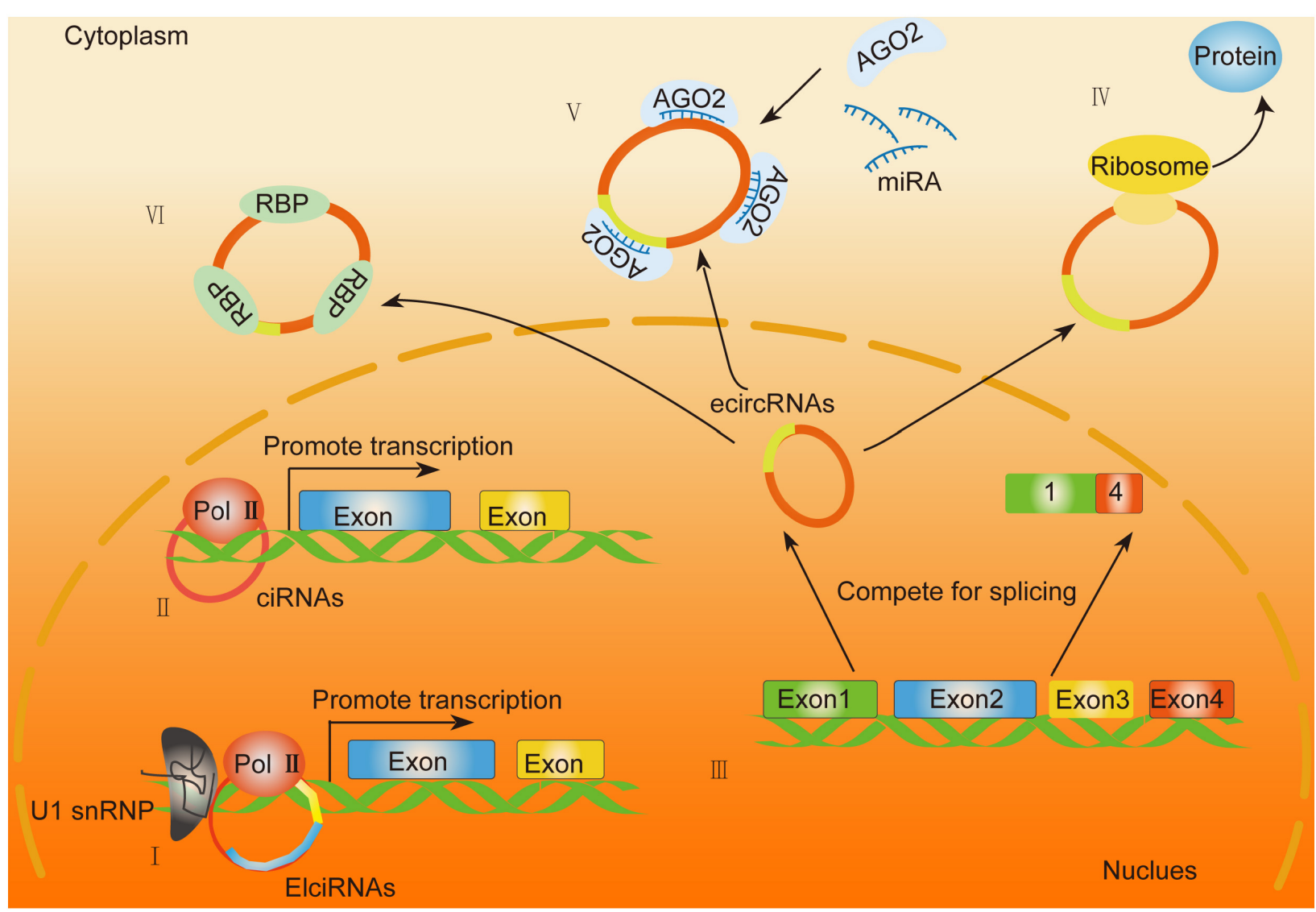

FIGURE 2 | General biological functions of circRNAs. (I,II) ElciRNAs and ciRNAs function as enhancers of specific proteins by binding with the RNA polymerase II (Pol II) complex. (III) EcircRNAs are generated through back-splicing and translocate from the nucleus into the cytoplasm. (IV) Exonic circRNAs that contain internal ribosome entry sites (IRES) or prokaryotic binding sites translate into peptides. (V) EcircRNAs function as miRNA sponges. (VI) EcircRNAs can act as a decoy or scaffold to sequester proteins and regulate gene expression or functional protein localization.

apoptosis through regulating $\mathrm{Wnt} / \beta$-catenin signal transduction, preventing the occurrence of HCC (Yang et al., 2020). Circ0051443, an exosomal circRNA, can transmit from normal cells to HCC cells via exosomes and suppress the malignant biological behaviors by promoting cell apoptosis and the cell cycle arrest (Chen W. et al., 2020). HCC is a typical type of cancer that is hypervascular. Endothelial cells have been confirmed to participate in angiogenesis and influence the development of HCC (Heimann et al., 1996). Circ_4911 and circ_4302 inhibits the formation of human umbilical vein endothelial cells (HUVECs) in the microenvironment of HCC (Yan et al., 2020). All these circRNAs have proven to have the potential to be therapeutic targets for HCC (Table 1). However, it is necessary to solve the limitation of the strategies that target them.

\section{CircRNAs Interfere With Hepatitis Virus Infection in HCC Progression}

Hepatitis virus infection is the primary cause of HCC. Chronic hepatitis B virus (HVB) infection takes the dominant risk factor in the majority of the areas of Asia and sub-Saharan Africa that have a high incidence of HCC (Cui et al., 2018).
The group of Ding has screened the circRNAs in HVB-related HCC patients by microarray. They have revealed 24 upregulated and 23 downregulated circRNAs significantly (fold-change $\geq 2$; $P \leq 0.05)$ in HCC tissues compared with non-tumorous tissues (NTs) (Cui et al., 2018). The top five upregulated circRNAs are hsa_circRNA_104351, hsa_circRNA_102814, hsa_circRNA_103489, hsa_circRNA_102109, and hsa_circ RNA_100381, and the top five downregulated circRNAs are hsa_circRNA_100327, hsa_circRNA_101764, hsa_circ RNA_101092, hsa_circRNA_001225, and hsa_circRNA_102904 (Cui et al., 2018). Among them, cRNA_101764 may play a dominant role in suppressing HCC progression through the PI3K-Akt signaling pathway (Wang et al., 2018).

\section{TRANSLATIONAL POTENTIAL OF CIRCRNA}

\section{CircRNAs Acting as Biomarkers}

The expression of circRNAs exhibits dynamic global changes during development (Jiao et al., 2020). For example, in humans, induction is observed across a variety of tissues and is consistently 
TABLE 2 | Representative circRNAs in the regulation of HCC.

\begin{tabular}{|c|c|c|}
\hline CircRNA & Function & Mechanism \\
\hline circRNA_100338 & Oncogene & $\begin{array}{l}\text { Function as a sponge of smiR-141-3p } \\
\text { (Huang et al., 2017). }\end{array}$ \\
\hline circ_0005075 & Oncogene & $\begin{array}{l}\text { Function as a sponge of miR- } 431 \text { (Li et al., } \\
\text { 2018). }\end{array}$ \\
\hline circ-ZNF652 & Oncogene & $\begin{array}{l}\text { Function as a sponge of } \\
\text { miR-29a-3p/GUCD1 (Li Y. et al., 2020). }\end{array}$ \\
\hline circMAST1 & Oncogene & $\begin{array}{l}\text { Function as a sponge of miRNA-1299 } \\
\text { Stabilizing CTNND1 (Yu X. et al., 2020). }\end{array}$ \\
\hline circZNF609 & Oncogene & $\begin{array}{l}\text { Function as a sponge of } \\
\text { miR-15a-5p/15b-5p Activating the } \\
\text { Hedgehog pathway (He et al., 2020). }\end{array}$ \\
\hline $\begin{array}{l}\text { exosome circ- } \\
\text { deubiquitination } \\
\text { (circ-DB) }\end{array}$ & Oncogene & $\begin{array}{l}\text { Function as a sponge of miR-34a Activating } \\
\text { deubiquitination-related USP7 (Zhang et al., } \\
\text { 2019). }\end{array}$ \\
\hline circ_0091579 & Oncogene & ND (Jiang et al., 2020) \\
\hline hsa_circ_0000711 & Oncogene & $\begin{array}{l}\text { Targeting has-miR-103a-3p (Chen K. H. } \\
\text { et al., 2020). }\end{array}$ \\
\hline Hsa_circ_104566 & Oncogene & $\begin{array}{l}\text { Decreasing apoptosis and E-cadherin (Liu } \\
\text { et al., 2020). }\end{array}$ \\
\hline circRNA-100338 & Oncogene & $\begin{array}{l}\text { Enhancing invasiveness and angiogenesis } \\
\text { (Huang et al., 2020). }\end{array}$ \\
\hline hsa_circ_0000092 & Oncogene & $\begin{array}{l}\text { hsa_circ_0000092 competitively bind to } \\
\text { miR-338-3p to up-regulate HN1 expression } \\
\text { (Pu et al., 2020). }\end{array}$ \\
\hline ciRS-7 (Cdr1as) & Oncogene & ND (Xu et al., 2017). \\
\hline circZKSCAN1 & $\begin{array}{l}\text { Tumor } \\
\text { suppressor }\end{array}$ & Inhibits Wnt signaling (Zhu et al., 2019). \\
\hline circ-MTO1 & $\begin{array}{l}\text { Tumor } \\
\text { suppressor }\end{array}$ & $\begin{array}{l}\text { Function as a sponge of miR-9 Promote } \\
\text { p21 expression (Han et al., 2017). }\end{array}$ \\
\hline cSMARCA5 & $\begin{array}{l}\text { Tumor } \\
\text { suppressor }\end{array}$ & $\begin{array}{l}\text { Function as a sponge of miR-17-3p } \\
\text { Function as a sponge of miR-181b-5p (Yu } \\
\text { et al., 2018). }\end{array}$ \\
\hline circ- 102,166 & $\begin{array}{l}\text { Tumor } \\
\text { suppressor }\end{array}$ & $\begin{array}{l}\text { Function as a sponge of miR-182 Function } \\
\text { as a sponge of miR-184 (Li R. et al., 2020). }\end{array}$ \\
\hline circRNA-ITCH & $\begin{array}{l}\text { Tumor } \\
\text { suppressor }\end{array}$ & $\begin{array}{l}\text { Regulating Wnt/ } / \beta \beta \text {-catenin signal } \\
\text { transduction (Yang et al., 2020). }\end{array}$ \\
\hline circ-0051443 & $\begin{array}{l}\text { Tumor } \\
\text { suppressor }\end{array}$ & $\begin{array}{l}\text { Promoting cell apoptosis and arresting the } \\
\text { cell cycle (Chen W. et al., 2020). }\end{array}$ \\
\hline Circ_4911 & $\begin{array}{l}\text { Tumor } \\
\text { suppressor }\end{array}$ & $\begin{array}{l}\text { Inhibits the formation of human umbilical } \\
\text { vein endothelial cells (HUVECs) (Yan et al., } \\
\text { 2020). }\end{array}$ \\
\hline circ_4302 & $\begin{array}{l}\text { Tumor } \\
\text { suppressor }\end{array}$ & $\begin{array}{l}\text { Inhibits the formation of human umbilical } \\
\text { vein endothelial cells (HUVECs) (Yan et al., } \\
\text { 2020). }\end{array}$ \\
\hline
\end{tabular}

observed for circRNAs spliced by both the major (U2) and minor (U12) spliceosome (Barrett and Salzman, 2016). This may be an induction that circRNAs exhibit a high degree of tissue specificity, and some correlates with the size of tumor, with the TNM stage, or metastasis (Zheng et al., 2019). Due to this specificity of circRNAs, they can be used as biomarkers for early cancer detection. Early detection of cancers has been a promising research focus globally. In the past years, circRNAs acting as biomarkers have attracted increasing interest for early detection of cancer. Several characteristics of circRNAs indicate the advantages of circRNAs as biomarkers. (a) Specific expression and high conservation: CircRNAs are expressed in a tissue/development stage-specific manner and most of them are conserved in variable species (Guo et al., 2014; Jeck and Sharpless, 2014; Wang et al., 2014; Zhang Y. G. et al., 2016); (b) stable structure: circRNAs are covalently closed loops, lacking of $5^{\prime}-3^{\prime}$ polarity and polyadenylated tails. Thus, circRNAs are resistant to RNase or RNA exonuclease activation (Zhang Y. G. et al., 2016), due to their higher stable structures compared with the linear RNAs (Suzuki and Tsukahara, 2014); and (c) high abundance: the abundance of circRNAs is comparable with its canonical linear transcripts (Salzman et al., 2012). CircRNAs can be detected in both tumor tissues and body fluids, including blood, saliva, and urine (Bahn et al., 2015; Li Y. et al., 2015; Vo et al., 2019). The specific circRNAs detected in body fluids would be useful indicators of cancer or diseases (Sand et al., 2016), which makes circRNAs ideal biomarkers for invasive detection.

\section{Potential of CircRNAs in HCC Therapy}

Although there are numerous reports of the functions of circRNAs in HCC, it is disappointing that there is no application of circRNAs in clinical cancer therapy directly. Only few of the researchers have tested and evaluated the potential application of targeting circRNAs in preclinical animal models. CircMYLK was determined to be significantly upregulated in HCC tissues and cells, and mouse tumorigenicity assay shows that injection of circMYLK small interfering RNA (siRNA) drastically suppresses xenograft tumor formation in vivo (Gao et al., 2020). Silencing circMAST1 with siRNA inhibits xenograft tumor migration, invasion, and proliferation in mouse (Yu X. et al., 2020). Recently, a group established a plasma circular RNA panel to diagnose hepatitis B virus-related HCC (Yu J. et al., 2020). Three plasma circRNAs are identified, namely hsa_circ_0000976, hsa_circ_0007750, and hsa_circ_0139897, which show higher accuracy than the serum biomarker alpha-fetoprotein (AFP) (Yu J. et al., 2020). This provides a guidance for HCC detection with serum circRNA. However, there are some limitations for this circpanel; for instance, all the HCC patients in this study were HBV-related, and further study of HCC caused by other factors should be evaluated. The application of circRNA in clinical treatment still needs a long way for development in the future.

\section{TRANSLATIONAL CHALLENGES AND PERSPECTIVES OF CIRCRNAS IN HCC}

CircRNAs have shown great potential as biomarkers for early cancer detection and as targets of cancer therapy (Fu et al., 2020; Sun et al., 2020; Zhu et al., 2021); however, some obstacles still need to be overcome. Firstly, the clinical relevance of circRNAs toward given cancers needs more mechanistic investigation and correlation analysis with a large cohort of patient samples to be confirmed. Secondly, technical improvement on the quantification of a specific circRNA and silencing it without affecting the expression of the parental linear transcript are in urgent need. Thirdly, as the detection hairpin probes should be designed to the back-splice junction sites, the clinical application 
of circRNAs is limited due to the lack of specific capture and detection in biopsy.

Recently, some research groups have established methods for the accurate quantification of circRNAs with an algorithm, CIRIquant, a consolidated computational pipeline, which helps unveil the regulation of competitive splicing between circRNAs and their linear counterparts (Wang et al., 2018; Di Liddo et al., 2019; Zhang et al., 2020). Zheng et al. have identified a new feature, reverse overlap (RO), for circRNA detection, which outperforms back-splice junction (BSJ)-based methods in identifying low-abundance circRNAs (Wang et al., 2018). Electrochemical detection of circRNAs combines back-splice junction recognition and duplex-specific nuclease-assisted target recycling signal amplification (Castañeda et al., 2017; Jiao et al., 2020). Due to its high sensitivity and reproducibility, it has been employed to assay circRNA in different concentrations into human $1 \%$ serum, $10 \%$ serum, and $10 \%$ peripheral blood to test the repeatability and stability of this method (Castañeda et al., 2017; Jiao et al., 2020). Even though these methods have shown excellent sensitivity, repeatability, and stability in experiments, but how about when these methods are applied in clinical detection? An experiment with large cohorts of HCC patients is needed for the evaluation of circRNA detection with these methodologies.

Moreover, the application of precise RNA interference (RNAi) to target oncogenic circRNAs in cancers should exclude the possibility of interference with the expression of cognate linear mRNAs. As for this purpose, the interference RNAs should be designed to accurately target the unique back-spliced junctions of oncogenic circRNAs. HCC is one of the most malignant cancers worldwide. There is a great need for the combination of interdisciplines to develop clinical tools for early detection and therapy of HCC. In the past few years, a plethora of studies have revealed that circRNAs are systematically altered

\section{REFERENCES}

Abdelmohsen, K., Panda, A. C., Munk, R., Grammatikakis, I., Dudekula, D. B., De, S., et al. (2017). Identification of HuR target circular RNAs uncovers suppression of PABPN1 translation by CircPABPN1. RNA Biol. 14, 361-369. doi: 10.1080/15476286.2017.1279788

Aktas, T., Avsar Ilik, I., Maticzka, D., Bhardwaj, V., Pessoa Rodrigues, C., Mittler, G., et al. (2017). DHX9 suppresses RNA processing defects originating from the Alu invasion of the human genome. Nature 544, 115-119. doi: 10.1038/ nature 21715

Andres-Leon, E., Nunez-Torres, R., and Rojas, A. M. (2016). miARma-Seq: a comprehensive tool for miRNA, mRNA and circRNA analysis. Sci. Rep. 6:25749. doi: 10.1038/srep25749

Ashwal-Fluss, R., Meyer, M., Pamudurti, N. R., Ivanov, A., Bartok, O., Hanan, M., et al. (2014). circRNA biogenesis competes with pre-mRNA splicing. Mol. Cell 56, 55-66. doi: 10.1016/j.molcel.2014.08.019

Bahn, J. H., Zhang, Q., Li, F., Chan, T. M., Lin, X., Kim, Y., et al. (2015). The landscape of microRNA, Piwi-interacting RNA, and circular RNA in human saliva. Clin. Chem. 61, 221-230. doi: 10.1373/clinchem.2014.230433

Barrett, S. P., and Salzman, J. (2016). Circular RNAs: analysis, expression and potential functions. Development 143, 1838-1847. doi: 10.1242/dev.128074

Bray, F., Ferlay, J., Soerjomataram, I., Siegel, R. L., Torre, L. A., and Jemal, A. (2018). Global cancer statistics 2018: GLOBOCAN estimates of incidence and mortality worldwide for 36 cancers in 185 countries. CA Cancer J. Clin. 68, 394-424. doi: $10.3322 /$ caac. 21492 in HCC. The characterization of the mechanisms by which these circRNAs contribute to cancer offers opportunities for the early diagnosis, evaluation of prognosis, and therapeutic intervention of HCC. There are lots of circRNAs identified to be involved in HCC tumor cell-autonomous processes, including cell proliferation, apoptosis, invasion, and metastasis, but the functions of circRNAs in the tumor microenvironment of HCC are limited (Chen Y. et al., 2020; Zhou et al., 2020), which needs further investigation. To date, most researches on circRNAs are identified based on HCC cell lines. It is worth noting that HCC has high heterogeneity, and it is not precise to draw a functional conclusion about a specific circRNA studied within limited HCC cell lines. Thus, it is urgent to develop tools to detect circRNAs with body fluid of patients. Although there is a long way to go for the clinical translation of circRNAs, the rapid advancement of technologies and increasing research in the area of circRNA will make the "dark world" of circRNAs enlightened.

\section{AUTHOR CONTRIBUTIONS}

JC and TX designed and instructed the structure of this manuscript. MeW and MiW wrote this manuscript and make the revisions.

\section{FUNDING}

This research was supported by grants from the Start-up Grant of HZNU (4125C5021820470), National Natural Science Foundation of China (81802338, 82072646, 81730108, and 81973635), Zhejiang Provincial Natural Science Foundation of China for Distinguished Young Scholars (LR21H160001) (to JC), and Zhejiang Xinmiao (New-Shoot) Talent Project of China (2019R426066) (to MeW).

Castañeda, A. D., Brenes, N. J., Kondajji, A., and Crooks, R. M. (2017). Detection of microRNA by electrocatalytic amplification: a general approach for singleparticle biosensing. J. Am. Chem. Soc. 139, 7657-7664. doi: 10.1021/jacs. $7 \mathrm{~b} 03648$

Chen, K. H., Pan, J. F., Chen, Z. X., Pan, D., Gao, T., Huang, M., et al. (2020). Effects of hsa_circ_0000711 expression level on proliferation and apoptosis of hepatoma cells. Eur. Rev. Med. Pharmacol. Sci. 24, 4161-4171. doi: 10.26355/ eurrev_202004_20996

Chen, W., Quan, Y., Fan, S., Wang, H., Liang, J., Huang, L., et al. (2020). Exosome-transmitted circular RNA hsa_circ_0051443 suppresses hepatocellular carcinoma progression. Cancer Lett. 475, 119-128. doi: 10.1016/ j.canlet.2020.01.022

Chen, Y., Li, Y., Zheng, G., and Zhou, P. (2020). Construction and analysis of macrophage infiltration related circRNA-miRNA-mRNA regulatory networks in hepatocellular carcinoma. PeerJ 8:e10198. doi: 10.7717/peerj.10198

Conn, S. J., Pillman, K. A., Toubia, J., Conn, V. M., Salmanidis, M., Phillips, C. A., et al. (2015). The RNA binding protein quaking regulates formation of circRNAs. Cell 160, 1125-1134. doi: 10.1016/j.cell.2015.02.014

Cui, S., Qian, Z., Chen, Y., Li, L., Li, P., and Ding, H. (2018). Screening of upand downregulation of circRNAs in HBV-related hepatocellular carcinoma by microarray. Oncol. Lett. 15, 423-432. doi: 10.3892/ol.2017.7265

Di Liddo, A., de Oliveira Freitas, Machado, C., Fischer, S., Ebersberger, S., Heumuller, A. W., et al. (2019). A combined computational pipeline to detect circular RNAs in human cancer cells under hypoxic stress. J. Mol. Cell Biol. 11, 829-844. doi: 10.1093/jmcb/mjz094 
Du, W. W., Yang, W., Liu, E., Yang, Z., Dhaliwal, P., and Yang, B. B. (2016). Foxo3 circular RNA retards cell cycle progression via forming ternary complexes with p21 and CDK2. Nucleic Acids Res. 44, 2846-2858. doi: 10.1093/nar/gkw027

Esteller, M. (2011). Non-coding RNAs in human disease. Nat. Rev. Genet. 12, 861-874. doi: 10.1038/nrg3074

Filippenkov, I. B., Kalinichenko, E. O., Limborska, S. A., and Dergunova, L. V. (2017). Circular RNAs-one of the enigmas of the brain. Neurogenetics 18, 1-6. doi: 10.1007/s10048-016-0490-4

Fu, L. Y., Wang, S. W., Hu, M. Y., Jiang, Z. L., Shen, L. L., Zhou, Y. P., et al. (2020). Circular RNAs in liver diseases: mechanisms and therapeutic targets. Life Sci. 264, 118707. doi: 10.1016/j.lfs.2020.118707

Gao, J., Li, E., Liu, W., Yang, Q., Xie, C., Ai, J., et al. (2020). Circular RNA MYLK promotes hepatocellular carcinoma progression through the miR29a/KMT5C signaling pathway. Onco Targets Ther. 13, 8615-8627. doi: 10.2147/OTT. S258715

Guo, J. U., Agarwal, V., Guo, H., and Bartel, D. P. (2014). Expanded identification and characterization of mammalian circular RNAs. Genome Biol. 15:409. doi: 10.1186/s13059-014-0409-z

Han, B., Chao, J., and Yao, H. (2018). Circular RNA and its mechanisms in disease: from the bench to the clinic. Pharmacol. Ther. 187, 31-44. doi: 10.1016/j. pharmthera.2018.01.010

Han, D., Li, J., Wang, H., Su, X., Hou, J., Gu, Y., et al. (2017). Circular RNA circMTO1 acts as the sponge of microRNA-9 to suppress hepatocellular carcinoma progression. Hepatology 66, 1151-1164. doi: 10.1002/hep.29270

Hansen, T. B., Jensen, T. I., Clausen, B. H., Bramsen, J. B., Finsen, B., Damgaard, C. K., et al. (2013). Natural RNA circles function as efficient microRNA sponges. Nature 495, 384-388. doi: 10.1038/nature11993

He, Y., Huang, H., Jin, L., Zhang, F., Zeng, M., Wei, L., et al. (2020). CircZNF609 enhances hepatocellular carcinoma cell proliferation, metastasis, and stemness by activating the Hedgehog pathway through the regulation of miR-15a5p/15b-5p and GLI2 expressions. Cell Death Dis. 11:358. doi: 10.1038/s41419020-2441-0

Heimann, R., Ferguson, D., Powers, C., Recant, W. M., Weichselbaum, R. R., and Hellman, S. (1996). Angiogenesis as a predictor of long-term survival for patients with node-negative breast cancer. J. Natl. Cancer Inst. 88, 1764-1769. doi: $10.1093 /$ jnci/88.23.1764

Hentze, M. W., and Preiss, T. (2013). Circular RNAs: splicing's enigma variations. EMBO J. 32, 923-925. doi: 10.1038/emboj.2013.53

Hsu, M. T., and Coca-Prados, M. (1979). Electron microscopic evidence for the circular form of RNA in the cytoplasm of eukaryotic cells. Nature 280, 339-340. doi: $10.1038 / 280339 \mathrm{a} 0$

Huang, X. Y., Huang, Z. L., Huang, J., Xu, B., Huang, X. Y., Xu, Y. H., et al. (2020). Exosomal circRNA-100338 promotes hepatocellular carcinoma metastasis via enhancing invasiveness and angiogenesis. J. Exp. Clin. Cancer Res. 39:20. doi: 10.1186/s13046-020-1529-9

Huang, X. Y., Huang, Z. L., Xu, Y. H., Zheng, Q., Chen, Z., Song, W., et al. (2017). Comprehensive circular RNA profiling reveals the regulatory role of the circRNA-100338/miR-141-3p pathway in hepatitis B-related hepatocellular carcinoma. Sci. Rep. 7:5428. doi: 10.1038/s41598-017-05432-8

Ivanov, A., Memczak, S., Wyler, E., Torti, F., Porath, H. T., Orejuela, M. R., et al. (2015). Analysis of intron sequences reveals hallmarks of circular RNA biogenesis in animals. Cell Rep. 10, 170-177. doi: 10.1016/j.celrep.2014.12.019

Jeck, W. R., and Sharpless, N. E. (2014). Detecting and characterizing circular RNAs. Nat. Biotechnol. 32, 453-461. doi: 10.1038/nbt.2890

Jeck, W. R., Sorrentino, J. A., Wang, K., Slevin, M. K., Burd, C. E., Liu, J., et al. (2013). Circular RNAs are abundant, conserved, and associated with ALU repeats. RNA 19, 141-157. doi: 10.1261/rna.035667.112

Jiang, P., Han, W., Fu, Y., and Chen, Q. (2020). The Hsa_circ_0091579/miR940/TACR1 axis regulates the development of hepatocellular carcinoma. Cancer Manage. Res. 12, 9087-9096. doi: 10.2147/CMAR.S259243

Jiao, J., Li, C., Ning, L., Shi, L., Wang, L., Xiang, Y., et al. (2020). Electrochemical detection of circRNAs based on the combination of back-splice junction and duplex-specific nuclease. Sens. Actuators B Chem. 302:127166. doi: 10.1016/j. snb.2019.127166

Kolakofsky, D. (1976). Isolation and characterization of Sendai virus DI-RNAs. Cell 8, 547-555. doi: 10.1016/0092-8674(76)90223-3

Legnini, I., Di Timoteo, G., Rossi, F., Morlando, M., Briganti, F., Sthandier, O., et al. (2017). Circ-ZNF609 is a circular RNA that can be translated and functions in myogenesis. Mol. Cell 66, 22. doi: 10.1016/j.molcel.2017. 02.017

Li, J., Sun, D., Pu, W., Wang, J., and Peng, Y. (2020). Circular RNAs in cancer: biogenesis, function, and clinical significance. Trends Cancer 6, 319-336. doi: 10.1016/j.trecan.2020.01.012

Li, M. F., Li, Y. H., He, Y. H., Wang, Q., Zhang, Y., Li, X. F., et al. (2018). Emerging roles of hsa_circ_0005075 targeting miR-431 in the progress of HCC. Biomed. Pharmacother. 99, 848-858. doi: 10.1016/j.biopha.2018.01.150

Li, R., Deng, Y., Liang, J., Hu, Z., Li, X., Liu, H., et al. (2020). Circular RNA circ102,166 acts as a sponge of miR-182 and miR-184 to suppress hepatocellular carcinoma proliferation and invasion. Cell. Oncol. doi: 10.1007/s13402-02000564-y [Epub ahead of print].

Li, Y., Zang, H., Zhang, X., and Huang, G. (2020). Exosomal Circ-ZNF652 promotes cell proliferation, migration, invasion and glycolysis in hepatocellular carcinoma via miR-29a-3p/GUCD1 axis. Cancer Manage. Res. 12, 7739-7751. doi: 10.2147/CMAR.S259424

Li, Y., Zheng, Q., Bao, C., Li, S., Guo, W., Zhao, J., et al. (2015). Circular RNA is enriched and stable in exosomes: a promising biomarker for cancer diagnosis. Cell Res. 25, 981-984. doi: 10.1038/cr.2015.82

Li, Z., Huang, C., Bao, C., Chen, L., Lin, M., Wang, X., et al. (2015). Exon-intron circular RNAs regulate transcription in the nucleus. Nat. Struct. Mol. Biol. 22, 256-264. doi: 10.1038/nsmb.2959

Liang, D., Tatomer, D. C., Luo, Z., Wu, H., Yang, L., Chen, L. L., et al. (2017). The output of protein-coding genes shifts to circular RNAs when the premRNA processing machinery is limiting. Mol. Cell 68, 940-954.e3. doi: 10.1016/ j.molcel.2017

Lim, T. B., Aliwarga, E., Luu, T. D. A., Li, Y. P., Ng, S. L., Annadoray, L., et al. (2019). Targeting the highly abundant circular RNA circSlc8al in cardiomyocytes attenuates pressure overload induced hypertrophy. Cardiovasc. Res. 115, 19982007. doi: $10.1093 / \mathrm{cvr} / \mathrm{cvz} 130$

Liu, G., Guo, W., Rao, M., Qin, J., Hu, F., and Li, K. (2020). circRNA hsa_circ_104566 sponged miR-338-3p to Promote Hepatocellular Carcinoma Progression. Cell Transplant. 29:963689720963948. doi: 10.1177/0963689720963948

Lu, Q., Liu, T., Feng, H., Yang, R., Zhao, X., Chen, W., et al. (2019). Circular RNA circSLC8A1 acts as a sponge of miR-130b/miR-494 in suppressing bladder cancer progression via regulating PTEN. Mol. Cancer 18:111. doi: 10.1186/ s12943-019-1040-0

Memczak, S., Jens, M., Elefsinioti, A., Torti, F., Krueger, J., Rybak, A., et al. (2013). Circular RNAs are a large class of animal RNAs with regulatory potency. Nature 495, 333-338. doi: 10.1038/nature11928

Noto, J. J., Schmidt, C. A., and Matera, A. G. (2017). Engineering and expressing circular RNAs via tRNA splicing. RNA Biol. 14, 978-984. doi: 10.1080/15476286. 2017.1317911

Pamudurti, N. R., Bartok, O., Jens, M., Ashwal-Fluss, R., Stottmeister, C., Ruhe, L., et al. (2017). Translation of CircRNAs. Mol. Cell 66, 9-21.e7. doi: 10.1016/j. molcel.2017.02.021

Pu, J., Wang, J., Li, W., Lu, Y., Wu, X., Long, X., et al. (2020). hsa_circ_0000092 promotes hepatocellular carcinoma progression through up-regulating HN1 expression by binding to microRNA-338-3p. J. Cell Mol. Med. doi: 10.1111/ jcmm.15010 [Epub ahead of print].

Qian, L., Yu, S., Chen, Z., Meng, Z., Huang, S., and Wang, P. (2018). The emerging role of circRNAs and their clinical significance in human cancers. Biochim. Biophys. Acta Rev. Cancer 1870, 247-260. doi: 10.1016/j.bbcan.2018.06.002

Salzman, J., Chen, R. E., Olsen, M. N., Wang, P. L., and Brown, P. O. (2013). Cell-type specific features of circular RNA expression. PLoS Genet. 9:e1003777. doi: 10.1371/journal.pgen.1003777

Salzman, J., Gawad, C., Wang, P. L., Lacayo, N., and Brown, P. O. (2012). Circular RNAs are the predominant transcript isoform from hundreds of human genes in diverse cell types. PLoS One 7:e30733. doi: 10.1371/journal.pone. 0030733

Sand, M., Bechara, F. G., Gambichler, T., Sand, D., Bromba, M., Hahn, S. A., et al. (2016). Circular RNA expression in cutaneous squamous cell carcinoma. J. Dermatol. Sci. 83, 210-218. doi: 10.1016/j.jdermsci.2016.05.012

Sanger, H. L., Klotz, G., Riesner, D., Gross, H. J., and Kleinschmidt, A. K. (1976). Viroids are single-stranded covalently closed circular RNA molecules existing as highly base-paired rod-like structures. Proc. Natl. Acad. Sci. U.S.A. 73, 3852-3856. doi: 10.1073/pnas.73.11.3852 
Satoh, J., and Yamamura, T. (2004). Gene expression profile following stable expression of the cellular prion protein. Cell. Mol. Neurobiol. 24, 793-814. doi: 10.1007/s10571-004-6920-0

Sun, J. Y., Zhang, X. Y., Cao, Y. Z., Zhou, X., Gu, J., and Mu, X. X. (2020). Diagnostic and prognostic value of circular RNAs in hepatocellular carcinoma. J. Cell. Mol. Med. 24, 5438-5445. doi: 10.1111/jcmm.15258

Suzuki, H., and Tsukahara, T. (2014). A view of pre-mRNA splicing from RNase R resistant RNAs. Int. J. Mol. Sci. 15, 9331-9342. doi: 10.3390/ijms15069331

Szabo, L., Morey, R., Palpant, N. J., Wang, P. L., Afari, N., Jiang, C., et al. (2016). Erratum to: statistically based splicing detection reveals neural enrichment and tissue-specific induction of circular RNA during human fetal development. Genome Biol. 17:263. doi: 10.1186/s13059-016-1123-9

Vadlamudi, Y., Dey, D. K., and Kang, S. C. (2020). Emerging Multicancer Regulatory Role of ESRP1: orchestration of alternative splicing to control EMT. Curr. Cancer Drug Targets 20, 654-665. doi: 10.2174/ 1568009620666200621153831

van Heesch, S., Witte, F., Schneider-Lunitz, V., Schulz, J. F., Adami, E., Faber, A. B., et al. (2019). The translational landscape of the human heart. Cell 178, 242-260.e29. doi: 10.1016/j.cell.2019.05.010

Vo, J. N., Cieslik, M., Zhang, Y., Shukla, S., Xiao, L., Zhang, Y., et al. (2019). The landscape of circular RNA in cancer. Cell 176, 869-881.e13. doi: 10.1016/j.cell. 2018.12.021

Wang, F., Nazarali, A. J., and Ji, S. (2016). Circular RNAs as potential biomarkers for cancer diagnosis and therapy. Am. J. Cancer Res. 6, 1167-1176.

Wang, P. L., Bao, Y., Yee, M. C., Barrett, S. P., Hogan, G. J., Olsen, M. N., et al. (2014). Circular RNA is expressed across the eukaryotic tree of life. PLoS One 9:e90859. doi: 10.1371/journal.pone.0090859

Wang, S., Cui, S., Zhao, W., Qian, Z., Liu, H., Chen, Y., et al. (2018). Screening and bioinformatics analysis of circular RNA expression profiles in hepatitis B-related hepatocellular carcinoma. Cancer Biomark. 22, 631-640. doi: 10.3233/ CBM- 170910

Xu, L., Zhang, M., Zheng, X., Yi, P., Lan, C., and Xu, M. (2017). The circular RNA ciRS-7 (Cdrlas) acts as a risk factor of hepatic microvascular invasion in hepatocellular carcinoma. J. Cancer Res. Clin. Oncol. 143, 17-27. doi: 10.1007/ s00432-016-2256-7

Yan, K., Cheng, W., Xu, X., Cao, G., Ji, Z., and Li, Y. (2020). Circulating RNAs, circ_4911 and circ_4302, are novel regulators of endothelial cell function under a hepatocellular carcinoma microenvironment. Oncol. Rep. 44, 1727-1735. doi: 10.3892/or.2020.7702

Yang, B., Zhao, J., Huo, T., Zhang, M., and Wu, X. (2020). Effects of CircRNAITCH on proliferation and apoptosis of hepatocellular carcinoma cells through inhibiting Wnt/beta-catenin signaling pathway. J. BUON 25, 1368-1374.

Yang, Q., Du, W. W., Wu, N., Yang, W., Awan, F. M., Fang, L., et al. (2017). A circular RNA promotes tumorigenesis by inducing c-myc nuclear translocation. Cell Death Differ. 24, 1609-1620. doi: 10.1038/cdd.2017.86

Yang, Y., Fan, X., Mao, M., Song, X., Wu, P., Zhang, Y., et al. (2017). Extensive translation of circular RNAs driven by N(6)-methyladenosine. Cell Res. 27, 626-641. doi: 10.1038/cr.2017.31

Yu, J., Ding, W. B., Wang, M. C., Guo, X. G., Xu, J., Xu, Q. G., et al. (2020). Plasma circular RNA panel to diagnose hepatitis B virus-related hepatocellular carcinoma: a large-scale, multicenter study. Int. J. Cancer 146, 1754-1763. doi: $10.1002 /$ ijc. 32647

Yu, J., Xu, Q. G., Wang, Z. G., Yang, Y., Zhang, L., Ma, J. Z., et al. (2018). Circular RNA cSMARCA5 inhibits growth and metastasis in hepatocellular carcinoma. J. Hepatol. 68, 1214-1227. doi: 10.1016/j.jhep.2018.01.012
Yu, X., Sheng, P., Sun, J., Zhao, X., Zhang, J., Li, Y., et al. (2020). The circular RNA circMAST1 promotes hepatocellular carcinoma cell proliferation and migration by sponging miR-1299 and regulating CTNND1 expression. Cell Death Dis. 11:340. doi: 10.1038/s41419-02 $0-2532-y$

Zeng, Y., Du, W. W., Wu, Y., Yang, Z., Awan, F. M., Li, X., et al. (2017). A Circular RNA binds to and activates AKT phosphorylation and nuclear localization reducing apoptosis and enhancing cardiac repair. Theranostics 7, 3842-3855. doi: 10.7150/thno.19764

Zhang, H., Deng, T., Ge, S., Liu, Y., Bai, M., Zhu, K., et al. (2019). Exosome circRNA secreted from adipocytes promotes the growth of hepatocellular carcinoma by targeting deubiquitination-related USP7. Oncogene 38, 2844-2859. doi: 10. 1038/s41388-018-0619-z

Zhang, J., Chen, S., Yang, J., and Zhao, F. (2020). Accurate quantification of circular RNAs identifies extensive circular isoform switching events. Nat. Commun. 11:90. doi: 10.1038/s41467-019-13840-9

Zhang, Y., Xue, W., Li, X., Zhang, J., Chen, S., Zhang, J. L., et al. (2016). The biogenesis of nascent circular RNAs. Cell Rep. 15, 611-624. doi: 10.1016/j.celrep. 2016.03.058

Zhang, Y., Zhang, X. O., Chen, T., Xiang, J. F., Yin, Q. F., Xing, Y. H., et al. (2013). Circular intronic long noncoding RNAs. Mol. Cell 51, 792-806. doi: 10.1016/j.molcel.2013.08.017

Zhang, Y. G., Yang, H. L., Long, Y., and Li, W. L. (2016). Circular RNA in blood corpuscles combined with plasma protein factor for early prediction of pre-eclampsia. BJOG 123, 2113-2118. doi: 10.1111/1471-0528.13897

Zheng, B., Wu, Z., Xue, S., Chen, H., Zhang, S., Zeng, T., et al. (2019). hsa_circRNA_100873 upregulation is associated with increased lymphatic metastasis of esophageal squamous cell carcinoma. Oncol. Lett. 18, 6836-6844. doi: 10.3892/ol.2019.11003

Zhou, H., Xu, Q., Ni, C., Ye, S., Xu, X., Hu, X., et al. (2018). Prospects of Noncoding RNAs in Hepatocellular Carcinoma. Biomed Res. Int. 2018, 6579436. doi: 10. 1155/2018/6579436

Zhou, P., Zheng, G., Li, Y., Wu, D., and Chen, Y. (2020). Construction of a circRNA-miRNA-mRNA network related to macrophage infiltration in hepatocellular carcinoma. Front. Genet. 11:1026. doi: 10.3389/fgene.2020. 01026

Zhu, J., Li, J., Wei, Y., Wang, J., and Zhang, X. Y. (2021). Roles of circular RNAs in the progression of hepatocellular carcinoma and their values as diagnostic and prognostic biomarkers. Gene 767:145175. doi: 10.1016/j.gene.2020. 145175

Zhu, Y. J., Zheng, B., Luo, G. J., Ma, X. K., Lu, X. Y., Lin, X. M., et al. (2019). Circular RNAs negatively regulate cancer stem cells by physically binding FMRP against CCAR1 complex in hepatocellular carcinoma. Theranostics 9, 3526-3540. doi: $10.7150 /$ thno. 32796

Conflict of Interest: The authors declare that the research was conducted in the absence of any commercial or financial relationships that could be construed as a potential conflict of interest.

Copyright (C) 2021 Wang, Wu, Xie and Chen. This is an open-access article distributed under the terms of the Creative Commons Attribution License (CC BY). The use, distribution or reproduction in other forums is permitted, provided the original author(s) and the copyright owner(s) are credited and that the original publication in this journal is cited, in accordance with accepted academic practice. No use, distribution or reproduction is permitted which does not comply with these terms. 